\section{A discrepancy between clinical course and magnetic resonance imaging in a case of non-her- petic acute limbic encephalitis}

\author{
Naoto Kohno, 1 Yuko Kawakami,2 \\ Chizuko Hamada,2 Genya Toyoda, 2 \\ Hirokazu Bokura,2 Shuhei Yamaguchi, 3 \\ Yukitoshi Takahashi4 \\ 1Department of Neurology, Ohda \\ Municipal Hospital, Ohda; 2 Department \\ of Neurology, Shimane Prefectural \\ Central Hospital, Izumo; ${ }^{3}$ Department of \\ Neurology, Faculty of Medicine, Shimane \\ University, Shimane; ${ }^{4}$ Department of \\ Research Shizuoka, National Epilepsy \\ Center, Shizuoka Institute of Epilepsy and \\ Neurological Disorders, Shizuoka, Japan
}

\section{Abstract}

We report the case of a 64-year old man who presented memory disturbance, low-grade fever, weight loss, and bilateral hand tremors for three months. He was diagnosed with nonherpetic acute limbic encephalitis (NHALE). Follow-up magnetic resonance imaging (MRI) revealed new lesions after symptomatic improvement following steroid pulse therapy. This may indicate that there is a time lag between the disturbance or recovery of neurons and astrocytes. Thus, other lesions might occasionally appear during convalescence in patients with NHALE, even if only minimal lesions were found on the initial MRI.

\section{Introduction}

Kusuhara reported 4 cases of acute encephalitis in which T2-weighted magnetic resonance imaging (MRI) of the brain showed high signal intensities in both the hippocampi and amygdaloid bodies. ${ }^{1}$ Polymerase chain reactions (PCR) for herpes simplex virus (HSV)-1 and HSV-2 DNA were negative, and paraneoplastic limbic encephalitis was excluded because of lack of malignancy. Fever and consciousness disturbances were found in all 4 cases, and convulsive seizures were reported in 3 . The consciousness disturbances cleared within ten days, but severe amnestic syndrome remained as sequelae. These cases were considered to be non-herpetic acute limbic encephalitis (NHALE). Steroid pulse therapy and gamma-globulin were effective against NHALE with autoantibodies against glutamate receptors (GluR) delta 2 and epsilon 2. Follow- up MRI showed that the abnormal findings in bilateral hippocampi and amygdaloid bodies decreased or disappeared during convalescence. ${ }^{2-5}$ We report a case of NHALE in which follow-up MRI revealed other lesions after symptomatic improvement by steroid therapy.

\section{Case Report}

A 64-year old man was admitted to our hospital for a memory disturbance. He had been treated for hypertension, hypercholesterolemia, gout, and gastroesophageal reflux disease, but he had no history of neurological or psychiatric illness. He occasionally drank alcohol. He had developed a $37-38^{\circ} \mathrm{C}$ low-grade fever and bilateral hand tremors three months previously and had lost $5 \mathrm{~kg}$ of body weight. He had become aware of the memory disturbance and his gait imbalance one month before admission. He showed no symptoms of focal infection such as a common cold or collagen disease, and hyperthyroidism was excluded. Laboratory data showed liver dysfunction and an inflammatory reaction. On admission, his body temperature was $36.9^{\circ} \mathrm{C}$; however, a physical examination of the chest and abdomen showed no abnormalities. His state of consciousness was E4, V4, and M6 on the Glasgow Coma Scale. He had no apparent aphasia and presented no abnormalities in the peripheral cranial nerves or hemiparesis of the extremities. Deep tendon reflexes were normal without a pathological reflex. Nuchal rigidity and Kernig's sign were absent. He had bilateral 7-8 $\mathrm{Hz}$ action tremors of the hands. There was no intention tremor in the lower limbs but his gait was unsteady. He was topographically disorientated and could never find his way to the restroom on the ward. Laboratory data, including a complete blood count, renal function, electrolytes, ammonia, thyroid hormones, antinuclear acid, cytoplasmic antineutrophil cytoplasmic antibody, perinuclear antineutrophil cytoplasmic antibody, matrix metalloproteinase-3, rheumatoid arthritis test, immune complex, cytomegalovirus antigenemia, and electrocardiography, were all normal. Liver dysfunction and C-reactive protein had been normalized. A cerebrospinal fluid (CSF) examination showed elevated cell counts, with monocyte levels of $70 \mu \mathrm{L}$ (polynuclear cells of 0 $\mu \mathrm{L})$. The CSF protein level was $67 \mathrm{mg} / \mathrm{dL}$. Diffusion-weighted imaging (DWI) and fluid attenuated inversion recovery (FLAIR) of his brain MRI on Day 2 showed hyperintensity in the bilateral medial temporal cortex (Figure 1A). Magnetic resonance angiography (MRA) showed no abnormalities (Figure 1B). N-isopropyl-[123I]p-iodoamphetamine single photon emission computed tomography (SPECT) showed hypoperfusion in the whole brain, pre-
Correspondence: Naoto Kohno, Department of Neurology, Ohda Municipal Hospital, Yoshinaga 1428-3, Ohda-cho, Ohda, 694-0063, Japan. Tel. +81.854.820330 - Fax: +81.854 .847749 . E-mail:kohnonaoto@gmail.com

Key words: non-herpetic acute limbic encephalitis, steroid pulse therapy, magnetic resonance imaging, autoantibodies against glutamate receptors epsilon 2 and delta 2 .

Conflict of interests: the authors declare no potential conflict of interests.

Received for publication: 17 April 2012

Revision received: 19 February 2013.

Accepted for publication: 20 March 2013

This work is licensed under a Creative Commons Attribution NonCommercial 3.0 License (CC BYNC 3.0).

(C) Copyright N. Kohno et al., 2013

Licensee PAGEPress, Italy

Neurology International 2013; 5:e8

doi:10.4081/ni.2013.e8

dominantly in the left frontal lobe (Figure 2). An electroencephalogram showed theta and delta waves in the frontotemporal regions bilaterally (Figure 3 ).

0n Day 3, a neuropsychiatric assessment gave the patient a score of 15 on the MiniMental State Examination (MMSE). He was disoriented about time and experienced difficulty of recall. But he could draw interlocking pentagons. He scored 12 points on the Frontal Assessment Battery (range 0-18, cut-off score 15), 682 points on the Kohs Block Design Test, and 32 out of 36 points on the Raven's Colored Progressive Matrices. The patient did not present consciousness disturbance or myelopathy, and the course of his illness was relatively slow. He did not seem to be suffering from acute disseminated encephalomyelitis. A clinical diagnosis of NHALE was made. He received $1000 \mathrm{mg}$ /day methylprednisolone intravenously from Day 2 to Day 4, followed by oral 50 $\mathrm{mg}$ /day prednisolone for three days, $40 \mathrm{mg}$ /day prednisolone for 14 days, $35 \mathrm{mg} /$ day prednisolone for seven days, $30 \mathrm{mg}$ /day prednisolone for seven days, and was taking $25 \mathrm{mg} /$ day prednisolone when he was discharged on Day 38 . He also received $5 \mathrm{~g} /$ day gamma globulin on Days 8 and 9, and 2.5 g gamma globulin on Day 10 . 0n Day 7, he only had a slight tremor of the hands and his gait was almost normal. On Day 10 , he was disorientated about time and place. On Day 12, he lost his way to the restroom on the ward. 0n Day 13, his disorientation improved. He could tell which month it was and when asked where he was could answer in hospital. After treatment, the clinical symptoms of memory disturbance, bilateral hand tremors, and unsteady gait gradually improved. 


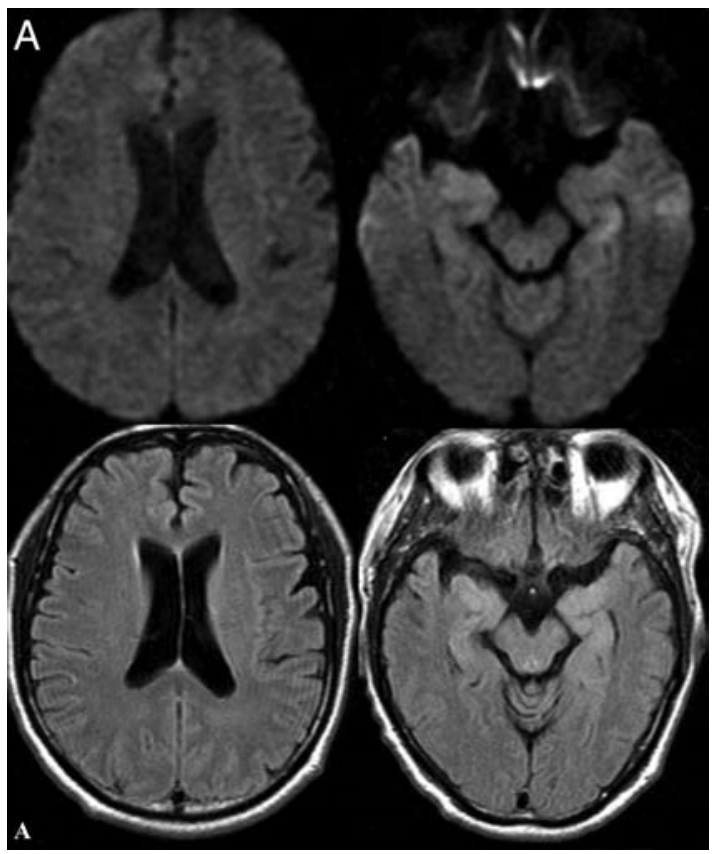

B

$\mathbf{R}$

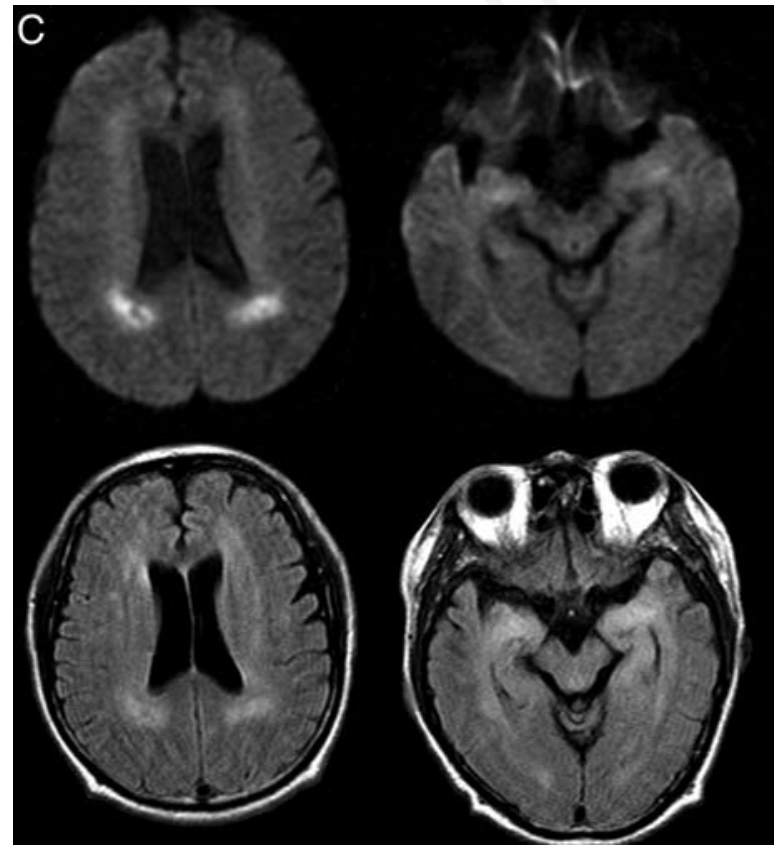

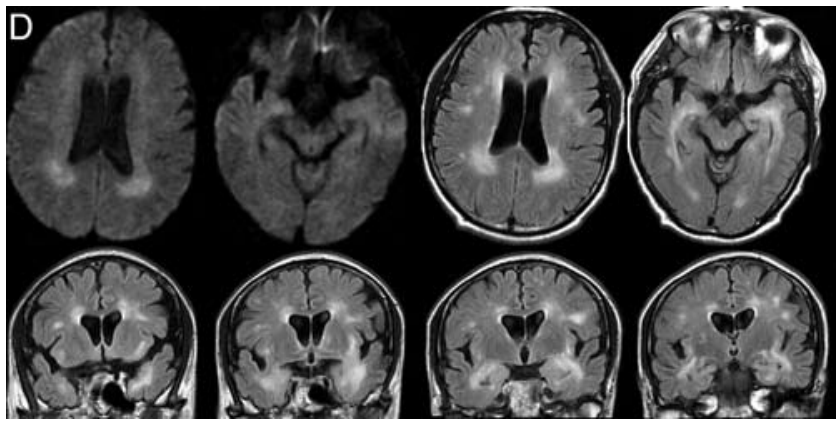

$\mathrm{E}$
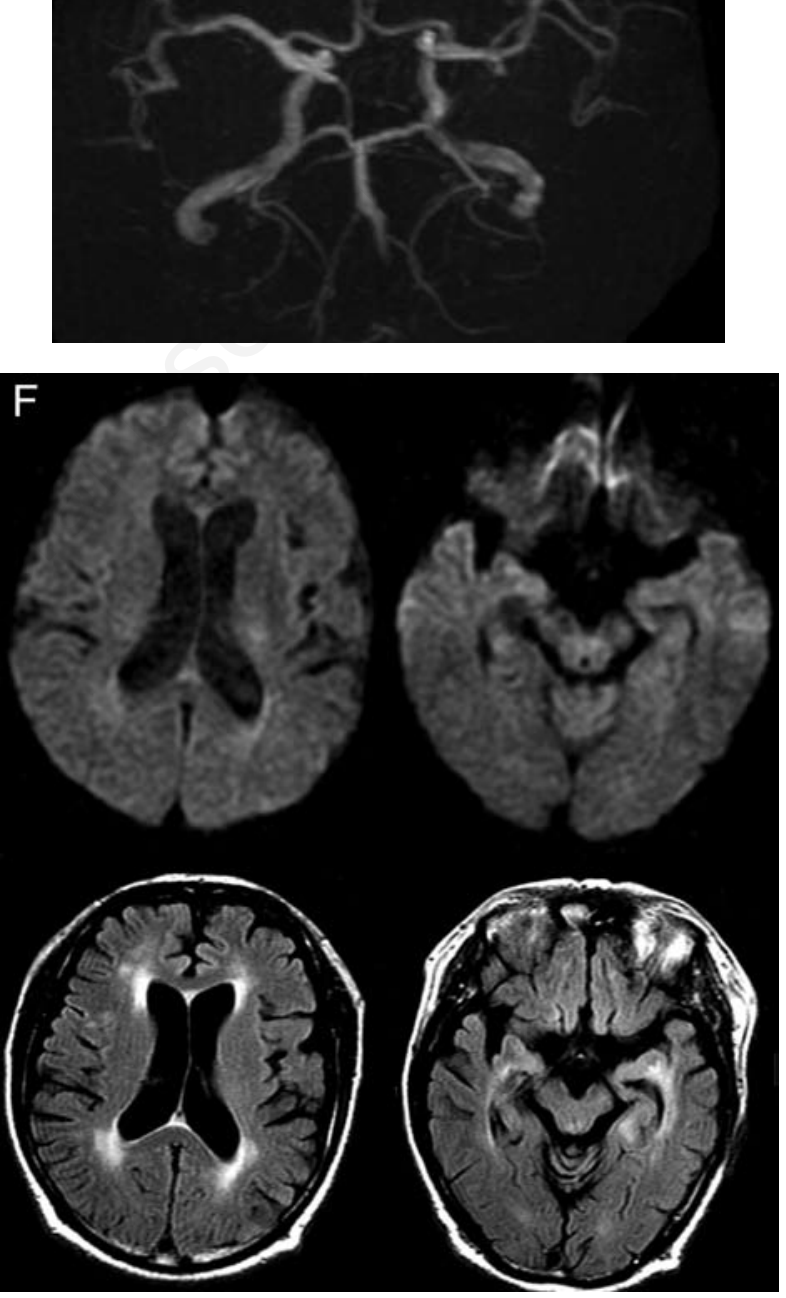

Figure 1. (A) Magnetic resonance imaging on Day 2 showed hyperintensity in the bilateral medial temporal cortex on diffusion-weighted imaging and fluid attenuated inversion recovery. (B) Magnetic resonance angiography showed no abnormalities. (C) Diffusion-weighted imaging and fluid attenuated inversion recovery on Day 14 revealed other hyperintense lesions around the lateral ventricles. (D) Diffusion-weighted imaging and FLAIR on Day 37 revealed that hyperintense lesions in the bilateral medial temporal cortex became more apparent in fluid attenuated inversion recovery image and lesions around the lateral ventricles remained. (E) Magnetic resonance angiography did not show any abnormal findings. (F) Magnetic resonance imaging 17 months later showed hyperintense lesions in the bilateral medial temporal cortex and on fluid attenuated inversion recovery the lesions around the lateral ventricles remained. 
He was eventually able to walk steadily in the ward without losing his way. But DWI and FLAIR on Day 14 revealed other hyperintense lesions around the lateral ventricles (Figure 1C). There have been reports of antibodies to $\mathrm{N}$-methyl-D-aspartate receptor (NMDAR), antibodies to NR2B- and NR2A-containing heteromers of the NMDAR detected by cellbased assay, ${ }^{7}$ antibodies against full-length GluR epsilon 2 (B18) and GluR delta 2 subunits confirmed by Western blot, 8 and antibodies against peptides of GluR subunit of NMDAR quantified by an enzyme-linked immunosorbent assay (ELISA). ${ }^{9}$ Using an ELISA, we examined serum and CSF antibodies against GluR epsilon 2, delta 2 and zeta 2. Serum and CSF antibodies against GluR epsilon 2-NT2, GluR epsilon 2-CT1, GluR delta 2-NT, GluR delta 2CT were positive. Serum and CSF antibodies against GluR epsilon 2-M3-4, GluR zeta 1-NT, GluR zeta 1-CT were negative; thus, the patient was diagnosed with NHALE. We did not look for the other antibodies that have been found in patients with autoimmune limbic encephalitis, i.e. using ELISA on HEK293 cell lysates ectopically expressing NR1 or NR1NR2B heteromers, ${ }^{10}$ NMDAR GluR epsilon 2 (NR2B, GluN2B) or GluR zeta 1 (NR1, GluN1) subunits in this study.11 Using PCR, we did not detect the DNA of HSV type 1, HSV type 2, and human herpes virus 6 in the CSF. Tumor markers, including carcinoembryonic antigen, carbohydrate antigen 19-9, and alpha-fetoprotein were within normal limits. Further examination with abdominal ultrasonography and enhanced computed tomography of the chest and abdomen revealed no abnormal findings.

A follow-up neuropsychiatric examination on Day 29 showed that the MMSE score had improved to 28 points. A neuropsychiatric examination on Day 37 revealed a full-scale intelligence quotient (IQ) score of 100 , a verbal IQ score of 100, and a performance IQ of 99 on the Wechsler Adult Intelligence Scale. But DWI and FLAIR on Day 37 revealed that hyperintense lesions in the bilateral medial temporal cortex became more apparent in the FLAIR image and there were still lesions around the lateral ventricles (Figure 1D). MRA did not show any abnormal findings (Figure 1E). The patient was discharged with little sequelae on Day 38. He was taking glimepiride for diabetes mellitus and atorvastatin calcium hydrate for hypercholestemia in addition to prednisolone when he was discharged.

A follow-up MRI performed 17 months later showed no abnormalities on DWI, but revealed that hyperintense lesions in the bilateral medial temporal cortex and around the lateral ventricles on FLAIR remained (Figure 1F). We had not performed a whole body positron emission tomography (PET) scan. But the patient had presented no symptoms of malignancy and his symptoms of encephalitis had not recured

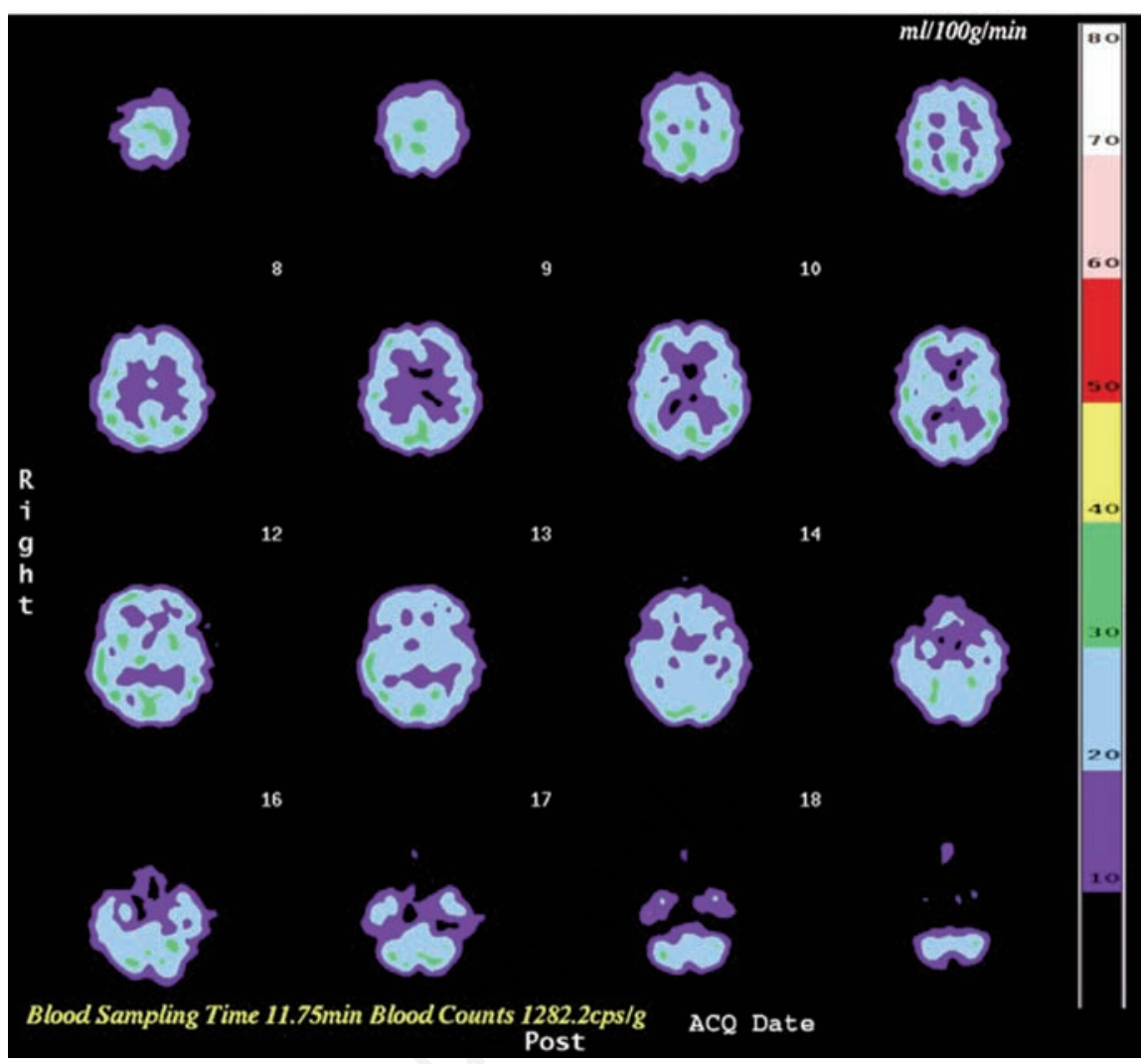

Figure 2. N-isopropyl-[123I]p-iodoamphetamine single photon emission computed tomography showed hypoperfusion in the whole brain, which was predominant in the left frontal lobe.

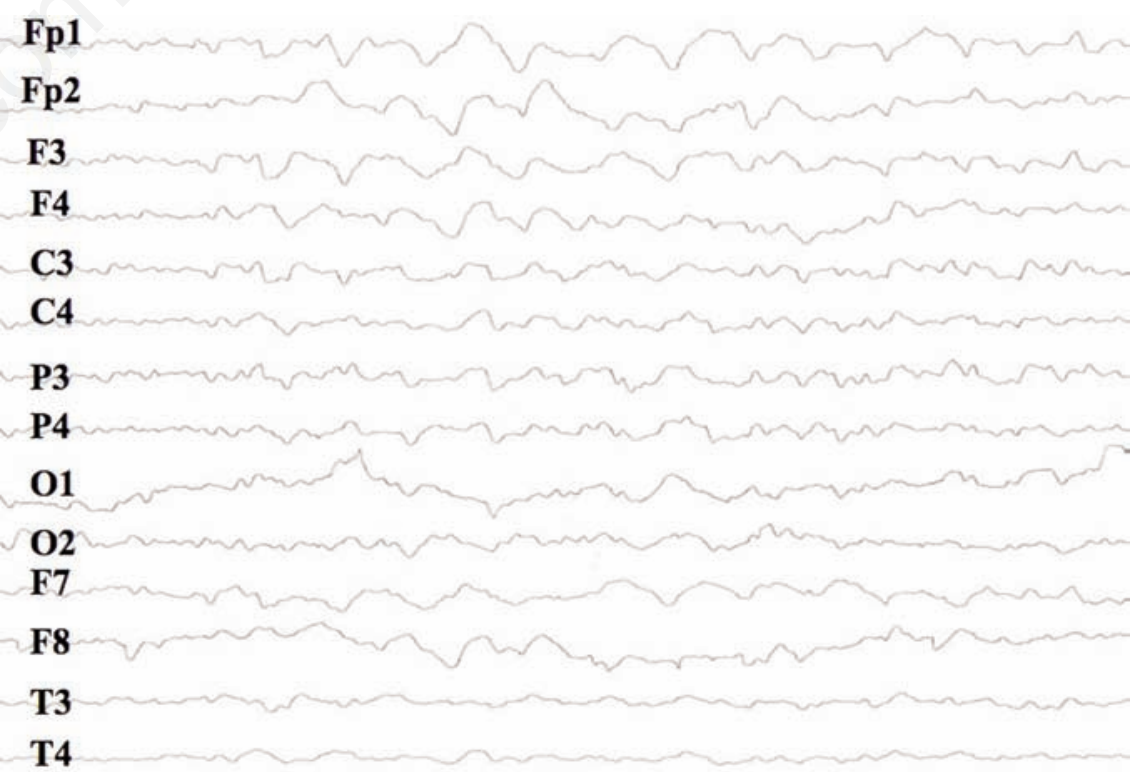

T5

T6

Figure 3. An electroencephalogram showed theta and delta waves in the frontotemporal regions bilaterally.

[Neurology International 2013; 5:e8]

[page 25] 
more than one year after he had stopped taking steroids. Thus, paraneoplastic limbic encephalitis has been ruled out.

\section{Discussion}

Takahashi reported 2 cases of NHALE and recognized four clinical features in both cases: i) an episode of preceding infection such as a common cold; ii) the appearance of reversible high signal intensity lesions in bilateral hippocampi and amygdaloid bodies on DWI; iii) elevation of only interleukin-6 in CSF; and iv) marked neurological improvement following intravenous administration of high-dose methylprednisolone. 4 Takahashi speculated that the immune reaction of the host might play a significant role in the pathogenesis of NHALE, based on these four clinical features. Our patient showed no symptoms of focal infection such as a common cold or collagen disease, and his low-grade fever might have been caused by NHALE.

Laboratory data in ambulant showed initial liver dysfunction and elevated C-reactive protein, but examination on admission showed that liver dysfunction and C-reactive protein had been normalized. The cause of the liver dysfunction was unknown, although a case of NHALE following a type- 2 adenovirus infection that presented high fever and severe liver dysfunction has been reported. 12

Steroid pulse therapy and gamma-globulin are effective against non-herpetic acute encephalitis with autoantibodies to GluR delta2 and epsilon2, and these antibodies in the CSF normalize during the clinical course.2,3 Intravenous and oral steroids, in addition to an intravenous gamma globulin infusion, are also effective against acute-onset non-neoplastic limbic encephalitis with hypogammaglobulinemia. ${ }^{13}$ Okamoto reported a case of paraneoplastic limbic encephalitis caused by an ovarian teratoma with autoantibodies to GluR in the CSF in which signs and symptoms improved following acyclovir and steroid pulse therapy. ${ }^{14}$ Symptoms of Hashimoto's encephalopathy with antibodies against the amino terminus of -enolase in the serum and those against GluR epsilon2 in the serum and CSF,15 as well as bilateral postural hand tremor, memory impairment, and insomnia caused by limbic encephalitis with the anti-voltage-gated potassium channel antibody, 16 also improved following steroid pulse therapy.

According to an MRI study in 91 adult patients with NHALE, DWI lesions were found in 20 of $49(40.8 \%)$ patients at $12.5 \pm 9.4$ days after onset, and FLAIR lesions were found in 32 of $59(54.2 \%)$ patients at $14.7 \pm 17.5$ days after onset.17 Follow-up MRI in convalescence showed abnormal findings in bilateral hippocampi and amygdaloid bodies that were reversed on DWI.4 T2-weighted and FLAIR imaging abnormalities decreased or disappeared followed by limbic atrophy during convalescence. ${ }^{5}$ A T1-weighted MRI three months after onset in a 31-year old woman with NHALE showed a linear high-signal intensity in the hippocampi, and the lesions seemed to be a focal necrosis. ${ }^{12}$ MRI abnormalities in children with NHALE are also reversible. 18,19 Takahashi et al. ${ }^{17}$ reported that MRI lesions appear in various stages after onset. However, we are unaware of any other case of NHALE in which a follow-up MRI disclosed other lesions during convalescence.

The neuropathological lesions in patients with NHALE were limited to the hippocampus and amygdala. The rostral portion of the hippocampus showed small foci characterized by neuronal loss with neuronophagia co-existing with proliferation of microglia, macrophages, hypertrophic astrocytes, and a few lymphocytes. The caudal portion of the hippocampus and amygdala showed neuronal loss with astrocytosis and lymphocytic perivascular cuffing. No leptomeningitis, hemorrhagic necrosis, or evidence of any etiological agent was detected microscopically. The abnormal high intensity areas seen on the MRI corresponded well with the astrocytosis regions. These findings are more similar to those in cases of autoimmune limbic encephalitis cases than herpes simplex encephalitis. The mild neuropathological changes seem to reflect a good clinical outcome.20

\section{Conclusions}

To our knowledge, this is the first published case of NHALE in which a follow-up MRI revealed that other lesions became more prominent after symptomatic improvement following steroid pulse therapy. The MRI findings of lesions in the temporal cortices were not compatible with those of demyelinating lesions. Although SPECT showed hypoperfusion in the whole brain, MR angiography did not show any abnormal findings on Day 2 or on Day 37. The lesions around the lateral ventricles were irreversible and it is not appropriate to consider them ischemic changes. The appearance of additional lesions after steroid therapy did not match the course of clinical symptoms and may indicate that there is a time lag between the disturbance or recovery of neurons and astrocytes. Thus, other lesions might appear occasionally during convalescence in patients with NHALE, even if only a few lesions are found on the initial MRI.

\section{References}

1. Kusuhara T, Shoji H, Kaji M, et al. [Nonherpetic acute limbic encephalitis]. Rinsho Shinkeigaku 1994;34:1083-8. [Article in Japanese].

2. Hayashi Y, Matsuyama Z, Takahashi Y, et al. [A case of non-herpetic acute encephalitis with autoantibodies for ionotropic glutamate receptor delta2 and epsilon2]. Rinsho Shinkeigaku 2005;45:657-62. [Article in Japanese].

3. Wada Y, Takahashi R, Yanagihara C, et al. [Successful use of methylprednisolone therapy in a case of non-herpetic acute encephalitis with antibodies against ionotropic glutamate receptor epsilon2 and delta2]. Brain Nerve 2007;59:527-32. [Article in Japanese].

4. Takahashi T, Kamei S, Miki K, et al. [The analysis of cytokines in cerebrospinal fluid (CSF) in two cases of non-herpetic acute limbic encephalitis (NHALE)]. Rinsho Shinkeigaku 2003;43:162-9. [Article in Japanese].

5. Asaoka K, Shoji H, Nishizaka S, et al. Nonherpetic acute limbic encephalitis: cerebrospinal fluid cytokines and magnetic resonance imaging findings. Intern Med 2004;43:42-8.

6. Terada T, Obi T, Miyajima H, Mizoguchi K. [Assessing frontal lobe function in patients with amyotrophic lateral sclerosis by frontal assessment battery]. Rinsho Shinkeigaku 2010;50:379-84. [Article in Japanese].

7. Dalmau J, Tüzün E, Wu HY, et al. Paraneoplastic anti-N-methyl-D-aspartate receptor encephalitis associated with ovarian teratoma. Ann Neurol 2007;61:25-36.

8. Takahashi Y, Mori H, Mishina M, et al. Autoantibodies to NMDA receptor in patients with chronic forms of epilepsia partialis continua. Neurology 2003;61:8916.

9. Fujita K, Yuasa T, Takahashi Y, et al. Antibodies to N-methyl-D-aspartate glutamate receptors in Creutzfeldt-Jakob disease patients. J Neuroimmunol 2012;251903 .

10. Dalmau J, Gleichman AJ, Hughes EG, et al. Anti-NMDA-receptor encephalitis: case series and analysis of the effects of antibodies. Lancet Neurol 2008;7:1091-8.

11. Takano S, Takahashi Y, Kishi H, et al. Detection of autoantibody against extracellular epitopes of $\mathrm{N}$-methyl-D-aspartate receptor by cell-based assay. Neurosci Res 2011;71:294-302.

12. Nagasawa H, Wada M, Kurita K, et al. [A case of non-herpetic acute limbic encephalitis associated with a type-2 adenovirus infection]. Rinsho Shinkeigaku 
2006;46:322-7. [Article in Japanese].

13. Akman CI, Patterson MC, Rubinstein A, Herzog R. Limbic encephalitis associated with anti-GAD antibody and common variable immune deficiency. Dev Med Child Neurol 2009;51:563-7.

14. Okamoto S, Hirano T, Takahashi Y, et al. Paraneoplastic limbic encephalitis caused by ovarian teratoma with autoantibodies to glutamate receptor. Intern Med 2007;46:1019-22.

15. Shindo A, Ii Y, Sasaki R, et al. [Non-herpetic acute limbic encephalitis-like manifestation in a case of Hashimoto's encephalopathy with positive autoantibodies against ionotropic glutamate receptor epsilon2] Rinsho Shinkeigaku 2007;47:629-34. [Article in Japanese].

16. Takado Y, Shimohata T, Tokunaga J, et al. [Patient with limbic encephalitis associated with anti-voltage-gated potassium channel antibodies who presented with insomnia and hand tremor]. Rinsho Shinkeigaku 2008;48:338-42. [Article in Japanese].

17. Takahashi Y, Kubota Y, Yamasaki E, Matsuda K. [Rasmussen encephalitis and non-herpetic acute limbic encephalitis]. Rinsho Shinkeigaku 2008;48:163-72. [Article in Japanese].
18. Kikuchi M, Watanabe S, Takahashi Y. [A case of non-herpetic acute limbic encephalitis in childhood--comparison with acute encephalitis with refractory repetitive partial seizures (AERRPS)]. No To Hattatsu 2007;39:221-5. [Article in Japanese].

19. Ishida $\mathrm{H}$, Hattori $\mathrm{H}$, Takaura $\mathrm{N}$, et al. [A child with non-herpetic acute limbic encephalitis affecting the claustrum and hippocampus]. No To Hattatsu 2006;38:443-7. [Article in Japanese].

20. Mochizuki Y, Mizutani T. [Neuropathological findings of non-herpetic limbic encephalitis]. Brain Nerve 2010;62:861-8. [Article in Japanese]. 